\title{
Research on Dynamic Target Distribution Path Planning Based on Improved Artificial Potential Field-Fish Algorithm in Logistics System
}

\author{
Qisong Zhanag ${ }^{1, a^{*}}$ and Peng Zhang ${ }^{2, b}$ \\ ${ }^{1}$ Dalian Neusoft Institute of Information, Dalian, China \\ ${ }^{2}$ Dalian JiaoTong University, Dalian, China \\ azhangqisong@neusoft.edu.cn, bzhangpeng@djtu.edu.cn
}

\begin{abstract}
Keywords: Improved potential field; Artificial fish swarm; Optimal path; Logistics distribution; Dynamic target
\end{abstract}

\begin{abstract}
Studied on path planning problem of logistics distribution vehicle in logistics system, and put forward an algorithm of artificial fish Swarm with improved potential field. The paper synthesizes variety of factors and takes the total cost of minimum distribution as goal in order to find out the optimal path between two points under known obstacle environment, and the algorithm meet the position change of user requirements in real time. Firstly, simulation experiments prove the algorithm effect in the logistics system; secondly, compared simple improved potential field algorithm, oil consumption and distance shorten significantly based on artificial fish swarm with potential field. The simulation result shows the new algorithm improves the searching efficiency.
\end{abstract}

\section{Introduction}

Logistics distribution is that the process of flow which is from supplier to recipient entity. In modern logistics system, the location of the accepting entity often changes, that needs delivery vehicles, real-time positioning position of the recipient entity to and deliver the goods precisely in the course of carriage. At the same time, delivery vehicles need some cost in the process of flow distribution. And in the fierce market competition environment, distribution enterprises in order to improve their own competitiveness, need to reduce the cost to a great extent. Logistics distribution problem is a NP problem, that needs intelligent algorithm to solve the problem .So this paper puts forward an improved artificial potential field-fish algorithm to solve the problem.

The paper studies the path planning problem in logistics system, especially for the more mature artificial potential field method, and proposes an improved artificial potential field method. Setting up artificial potential field under the environment of known obstacles, accepting entity generates "gravitational potential field", attracting delivery vehicle to move to itself. At the same time, the velocity vector is added in the traditional gravitational field function, that can make the delivery vehicle real-time positioning and track the position of recipient entity .Obstacles generate "repulsion potential field", distribution vehicle is rejected to move itself. Finally, under the action of two kinds of force, work out a distribution route. But the distribution route that is worked out from improved artificial potential field is not optimal path, the distribution route does not take into account factors that fuel consumption and distance of delivery vehicle. So this paper introduces into the artificial fish algorithm bas on artificial potential field, which can make evaluate and correct, and the optimal path is obtained.

\section{Improved Artificial Potential Field Method}

The basic ideas of traditional artificial potential field method is putting delivery vehicle in the known environment, and designs an abstract of artificial potential field. Accepting entity generates "gravity" for delivery vehicles, and obstacles generates "repulsion" for delivery vehicles. At last, resultant force is constituted by "gravity" and "repulsion" force that controls the distribution of the vehicle's 
movement. Working out the path based on artificial potential field is generally smooth and safe, that can make delivery vehicle to avoid obstacles and reach its target successfully.

The traditional artificial potential field is not suitable for dynamic target localization and tracking, and its gravitational field function is as follows:

$$
U_{\text {att }}(q)=\frac{1}{2} \xi \rho^{m}\left(q, q_{\text {goal }}\right)
$$

Among them, $q=[x, y]^{T}$ is current position for delivery vehicles. $q_{\text {goal }}=\left[x_{\text {goal }}, y_{\text {goal }}\right]^{T}$ is the position for recipient entity. $\xi$ is proportion coefficient $. \rho\left(q, q_{\text {goal }}\right)=\left\|q-q_{\text {goal }}\right\|$ is the distance between $q$ that the current position of delivery vehicle and $q_{\text {goal }}$ that target location, and $m=1$ or 2 .

It's not hard to see the gravitational field function of traditional artificial potential field method is only related to the position of delivery vehicles and recipient entity, that can not track dynamic target .

So this paper puts forward to a new gravitational field function based on a dynamic movement target basis the traditional artificial potential field method .In the gravitational field function, the velocity vector is added, so the delivery vehicle can be localization and tracking in dynamic environment smoothly, at the same time, under the repulsive force function, the user can effectively avoid obstacles.For illustration purposes, the following assumptions:

Assumption 1: user's location $q$ and speed $v$ is known.

Assumption 2: dynamic target's position $q_{\text {tar }}$ and velocity $v_{t a r}$ is known.

This paper introduces the velocity vector to the gravitational field function and puts forward a new kind of gravitational field function. The expression as shown in Eq.2:

$$
U_{a t t(q, v)}=\alpha_{q}\left\|q_{\operatorname{tar}}(t)-q(t)\right\|^{m}+\alpha_{v}\left\|v_{\operatorname{tar}}(t)-v(t)\right\|^{n}
$$

Among them, $q(t)$ and $q_{\mathrm{tar}}(t)$ are the position of the user and the moving targets at ${ }^{t}$ moment. $v(t)$ and $v_{\text {tar }}(t)$ velocity vector of user and the moving target's at $t$ moment. $\left\|\mathrm{q}_{\mathrm{tar}}(\mathrm{t})-\mathrm{q}(\mathrm{t})\right\|$ is the relative distance between the user and the moving target at $t$ moment of, $\left\|\mathrm{v}_{\operatorname{tar}}(t)-v(t)\right\|$ is the relative speed of the user and of moving target at $t$ moment. $\alpha_{q}$ and $\alpha_{v}$ are direct ratio coefficient. $m$ and $n$ are positive constants.

It's easy to judge from Eq.2, when velocity vector is added in the gravitational field function, if and only if the relative distance and speed of user and the dynamic target are zero, the function value is minimum value 0 .New gravitational field function increases as relative distance and relative speed of user and the target increases. The algorith can effectively locate and track the dynamic target.

\section{Artificial Fish Algorithm}

Artificial fish algorithm is a optimization algorithm that is a kind of intelligent simulation of the fish feeding behavior structure of multipoint parallel search .Artificial fish algorithm needs less specific information, not easy to fall into the locally optimal solution, thus it is applied in many fields .

Firstly, this paper uses improved artificial potential field method to generate the path for the dynamic target; secondly, uses artificial fish algorithm to evaluate the generated path, through the behavior of artificial fish, such as foraging, bunching, chase, to calculate the improved optimal path .

Design of Single Artificial Fish. For designing algorithm better, using intuitive, physical significance of artificial potential field parameters to construct a single fish. Choose direct proportion coefficient $\alpha_{\mathrm{q}}, \alpha_{\mathrm{v}}$, affect the distance $\rho_{0}$, the user's step length 1 and the repulsive force function of direct proportion coefficient $\varepsilon$ as a single code base of artificial fish. The form as shown below:

$$
x_{k}=\left(\alpha_{q}^{k}, \alpha_{v}^{k}, \rho_{0}^{k}, l^{k}, \varepsilon^{k}\right)
$$


Among them, $k$ is the number of artificial fish.

Fitness Function. Artificial fish algorithm by calculating the fitness function of the each of the artificial fish, pledges to fitness high with high probability to enter the next iteration, resulting in a higher fitness of artificial fish. This paper integrates distribution vehicle fuel consumption and mobility as factor of artificial fish fitness function, at the same time, according to the needs of movement to weigh.

1) Fuel Consumption $C_{r}$

$$
C_{r}=\varphi_{r} \sum_{j=1}^{N} L_{j}
$$

Among them, $\varphi_{\mathrm{r}}$ is fue consumption coefficient, $L_{j}$ the length of the route distance, $N$ steps for the user.

2)Maneuverability Cost

To correct the path in the logistics system, the number of correcting is the fewer, the path is more smoothly, the less resources is need.

$$
C_{m}=\mu_{m} \times N_{m}
$$

Among them, $\mu_{\mathrm{m}}$ is Maneuverability cost coefficient, and $\mathrm{N}_{\mathrm{m}}$ is the number of fixed path line.

In addition to the above two factors, but also can consider the arrival time, no-go areas and other factors. These factors can be according to the actual situation, carry on the corresponding increase or decrease. This paper will boil down the above factors to $C_{o}$, so the fitness function is expressed as:

$$
C_{\text {sum }}=\min \left(C_{r}+C_{m}+C_{o}\right)
$$

Revised Evaluation Criteria Based on the Path of Artificial Fish Algorithm. For path correction of evaluation include the following:

1) The total distance of path $(\mathrm{L})$, the path must be no greater than a preset maximum distance, this setting to ensure users can track the target in a fixed time.

2) The number of correct path number $(\mathrm{N})$, in order to reduce resource utilization, and set the number of correct path is no more than the maximum times.

3) Maximum turning Angle $(\theta)$, the generated path can only generate the Angle that is no greater than scheduled to determine the Angle ,ant angle usually limited in the range .That ensure smooth path.

When the generated path does not satisfy the above constraints, it needs to modify the path.

\section{Implementation Steps of Artificial Potential Field-Fish Algorithm}

Algorithm steps are as follows:

1) By improved artificial potential field method, calculate the navigation path.

2) Choose segmentation points from the angle of turning that are bigger, then use the least squares method of linear to fit paragraphs.

3) Calculate point of intersection which are from the adjacent fitting line segment, and connect starting point, point of intersection in line and dynamic target, generating the fitting path, namely the revised path

4) whether the path in line with the 3.3 line constraint, if meet the constraint condition, using the generated fixed path, or determining the segmentation point again, linear fitting again, and by increasing or decreasing the turning point to modify the path, until meet the constraint conditions.

\section{Simulation Results}

In order to verify the improved artificial potential field-fish effectiveness, this paper uses Visual C++ and Matlab to simulation experiment. In simulation system, the obstacles and the target position is 
artificial, step length of delivery vehicle is $0.5 \mathrm{~cm}$, and each time interval is $100 \mathrm{~ms}$. In order to simplify the research the experiment sets delivery vehicle and accepting entity are in constant speed motion. This paper compares the experiments to verify improved artificial potential field-fish algorithm superiority, Fig. 1 shows the improved artificial potential field - fish algorithm, and Fig. 2 shows improved artificial potential field algorithm. Two simulation diagram, green dot for delivery vehicles, starting position which is in left bottom of the simulation figure, tracking logistics entity for the black dot, in the upper left corner which is in same constant speed to right bottom corner , obstacles are artificial in the middle of diagram . Simulation results are as follows.

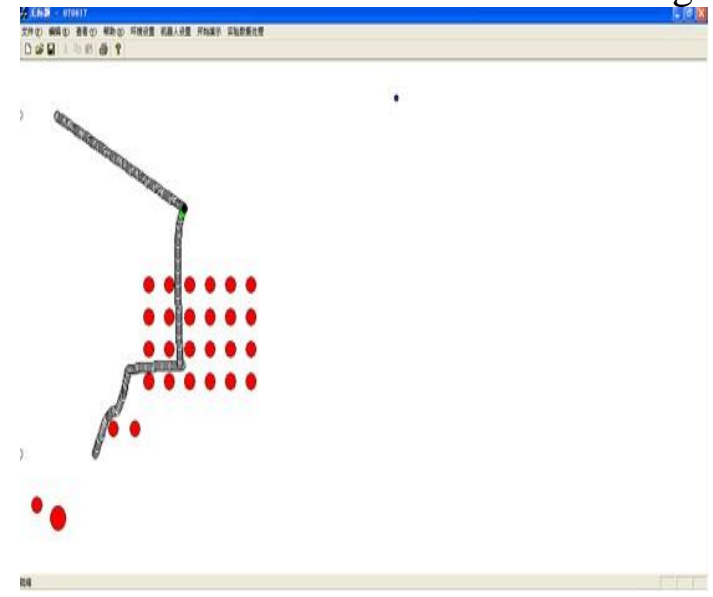

Figure 1. Improved artificial potential

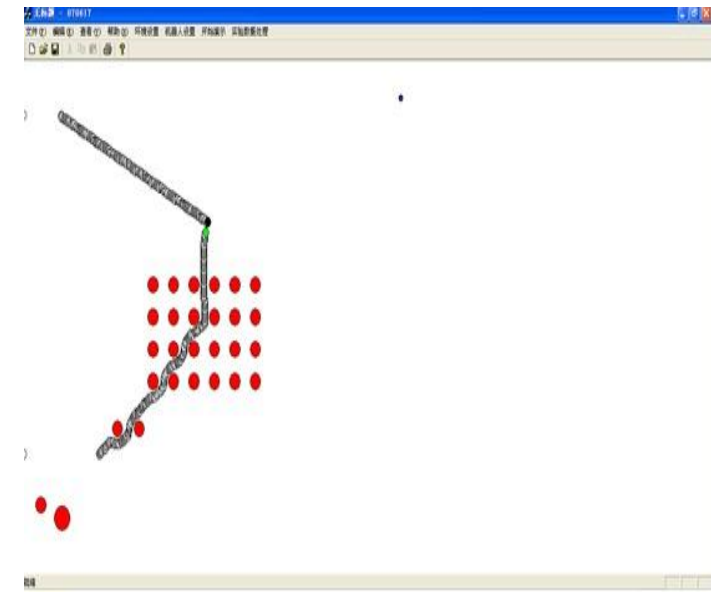

Figure 2. Improved artificial potential

Field-fish algorithm

Field algorithm

Through comparing simulation results above, firstly, demonstrate the effectiveness of the improved artificial potential field method, the new potential field function of resultant force can lead delivery vehicle avoid obstacles and track to mobile users. Secondly, by comparison, prove that the improved artificial potential field-fish algorithm generate the path that is shorter and the time is less.

To prove this algorithm's efficiency, this paper compares artificial potential field - fish algorithm and improved artificial potential field algorithm in the same environment. Horizontal axis is showed steps (oil consumption), vertical axis is showed the delivery time, the red line represents the improved artificial potential field-fish algorithm, blue line represents improved artificial potential field algorithm. Simulation results are as follows:

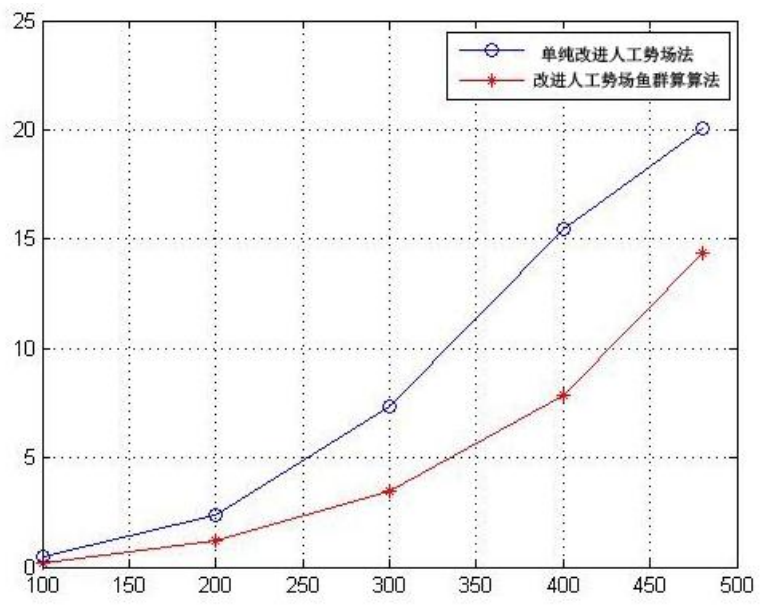

Figure 3. Contrast test

Experiments show that under the same experimental conditions, improved artificial potential field-fish algorithm and improved artificial potential field algorithm can search the valid path, but the 
steps and fuel consumption of improved artificial potential field-fish algorithm are less than improved artificial potential field algorithm. Illustrate the artificial potential field-fish algorithm is effective, and significantly improve the efficiency of path search the optimal path.

\section{Conclusions}

The improved artificial potential field- fish algorithm is proposed in this paper, and is applied to the path planning problem in the logistics system. In this paper, on the basis of the improved artificial potential field algorithm combined with artificial fish algorithm, use the algorithm to evaluate correct and obtain the optimal path. Finally, the simulation experiments show that the proposed algorithm has high practicability and validity.

\section{Acknowledgements}

Liaoning Provincial Natural Science Foundation (item number: 2014020170)

\section{References}

[1] Weihan Zhang, Yongjian Yang and Yu Zong, Study of the Algorithm of Optimal Route Navigation Based on LBS and Its Application: Journal of Hefei University of Technology, Vol.32(2009) No. 11,1744-1748.

[2] Maurizio Marchese, An Ant Colony Optimization Method for Generalized TSP Problem: Progress in Natural Science, Vol.11 (2008), 1417-1422.

[3] LEE Heow Pueh, LIM Siak Piang and LEE Kwok Hong, Solving traveling salesman problems by genetic algorithms: Progress in Natural Science, Vol. 02(2010), 57-63.

[4] Chih-Wei Liao, Jiun-Hung Yu and Yeong-Shin Tarng, On-line full scan inspection of particle size and shape using digital image processing:Particuology,Vol.03(2011),286-292.

[5] Wulan Huang and Tao Zhang, Vehicle Routing Problem with Simultaneous Pick-up and Delivery and Time-windows Based on Improved Global Artificial Fish Swarm Algorithm: Computer Engineering and Applications, 2016, Vol.52 (2016) No.21, 21-29.

[6] Tao Zhang, Chaovalitwongse W A and Yuejie Zhang, Integrated Ant Colony and Tabu Search Approach for Time Dependent Vehicle Routing Problems with Simultaneous Pickup and Delivery: Journal of Combinatorial Optimization, Vol.28 (2014), 28: 288-309.

[7] Wang H F and Chen Y Y,A genetic Algorithm for the Simultaneous Delivery and Pickup Problems with Time Window: Computers \& Industrial Engineering, Vol.62 (2012) No.1

[8] Azad M A K, A Simplified Binary Artificial Fish Swarm Algorithm for 0-1 Quadratic Knapsack Problems: Journal of Computational and Applied Mathematics, Vol.259 (2014), 897-904.

[9] Azad M A K, Improved Binary Artificial Fish Swarm Algorithm for the 0-1 Multidimensional Knapsack Problems: Swarm and Evolutionary Computation, Vol.14 (2014), 66-75.

[10]Fei Gao, Matlab Intelligent algorithm (Posts \& Telecom Press, China 2014) 